Article

\title{
Some Properties and Generating Functions of Generalized Harmonic Numbers
}

\author{
Giuseppe Dattoli ${ }^{1}$, Silvia Licciardi ${ }^{1} * *$ (D) Elio Sabia ${ }^{1}$ and Hari M. Srivastava ${ }^{2,3}$ \\ 1 ENEA-Frascati Research Center, Via Enrico Fermi 45, 00044 Frascati, Rome, Italy \\ 2 Department of Mathematics and Statistics, University of Victoria, Victoria, BC V8W 3R4, Canada \\ 3 Department of Medical Research, China Medical University Hospital, China Medical University, \\ Taichung 40402, Taiwan \\ * Correspondence: silviakant@gmail.com or silvia.licciardi@enea.it; Tel.: +39-392-509-6741
}

Received: 5 May 2019; Accepted: 24 June 2019; Published: 28 Junbe

check for updates

\begin{abstract}
In this paper, we introduce higher-order harmonic numbers and derive their relevant properties and generating functions by using an umbral-type method. We discuss the link with recent works on the subject, and show that the combinations of umbral and other techniques (such as the Laplace and other types of integral transforms) yield a very efficient tool to explore the properties of these numbers.
\end{abstract}

Keywords: umbral methods; harmonic numbers; special functions; integral representations; laplace and other integral transforms

MSC: 05A40, 44A99, 47B99; 11A99; 33C52, 33C65, 33C99, 33B10, 33B15; 44A05, 44A20; 44A10, 44A15

\section{Introduction}

The properties of many families of special numbers have been profitably studied by the use of methods tracing back to the umbral calculus [1]. Within this context, the definition of the associated polynomials naturally emerges as umbral Newton binomial convolutions (see "The Bernoulli Polynomials §4.2.2" in Reference [1]). The formalism is extremely powerful, and has allowed for the extension of the method to generalized forms of special numbers $([2,3])$. The use of umbral techniques has been recently employed in the study of harmonic numbers, whose relationship to Bernoulli numbers has been pointed out in Reference [4]. In this paper, we will extend the use of umbral methods to the case of higher-order harmonic numbers.

In a number of previous papers ([5-7]), different problems concerning harmonic numbers and the relevant generating functions have been touched. The already mentioned use of the umbral-like formalism has allowed for the framing of the theory of harmonic numbers within an algebraic context. Some of the points raised in ([5-7]) have been reconsidered, made rigorous, and generalized by means of different technical frameworks in successive research ([8-15]).

The present investigation concerns the application of the method foreseen in ([5-7]) to generalized forms of harmonic numbers, such as (We use the notation ${ }_{m} h_{n}$ instead of $H_{n}^{(m)}$ recommended in Reference [4] for continuity with previous papers, where it has been adopted to avoid confusion with higher-order Hermite polynomials):

$$
\begin{aligned}
& { }_{m} h_{n}=\sum_{r=1}^{n} \frac{1}{r^{m}}, \quad n \geq 1, \\
& { }_{m} h_{0}=0,
\end{aligned}
$$


namely, "higher-order harmonic numbers" satisfying the property:

$$
{ }_{m} h_{n+1}={ }_{m} h_{n}+\frac{1}{(n+1)^{m}},
$$

whose associated series is provided by the $\operatorname{limit}_{n \rightarrow \infty} \lim _{n} h_{n}, m>1$ is, unlike the ordinary harmonic numbers $(m=1)$, not diverging.

It can be argued that for negative $m$ values, the Harmonic numbers reduce to a finite sum of integers, expressible in terms of Bernoulli numbers, as discussed in the concluding part of the paper (Remark 3). In the following, we will derive a number of apparently new properties and the relevant consequences.

As an introductory example, we provide the following:

Example 1. We consider the second-order harmonic numbers $(m=2)$ and write

$$
{ }_{2} h_{n}=\int_{0}^{1} \frac{1-x^{n}}{x-1} \ln (x) d x, \quad \forall n \in \mathbb{N},
$$

which is obtained after setting

$$
\frac{1}{r^{2}}=\int_{0}^{\infty} e^{-s r} s d s
$$

by noting that

$$
{ }_{2} h_{n}=\int_{0}^{\infty} \frac{e^{-s(n+1)}-e^{-s}}{e^{-s}-1} s d s,
$$

and then by changing the variable of integration.

It is worth stressing that the integral representation allows for the extension of harmonic numbers to non-integer values of the index. The second-order harmonic numbers interpolates between the integer and real values of the index, as shown in the plot given in Figure 1, where it is pointed out that the asymptotic limit of the second-order harmonic numbers is $\frac{\pi^{2}}{6}$.

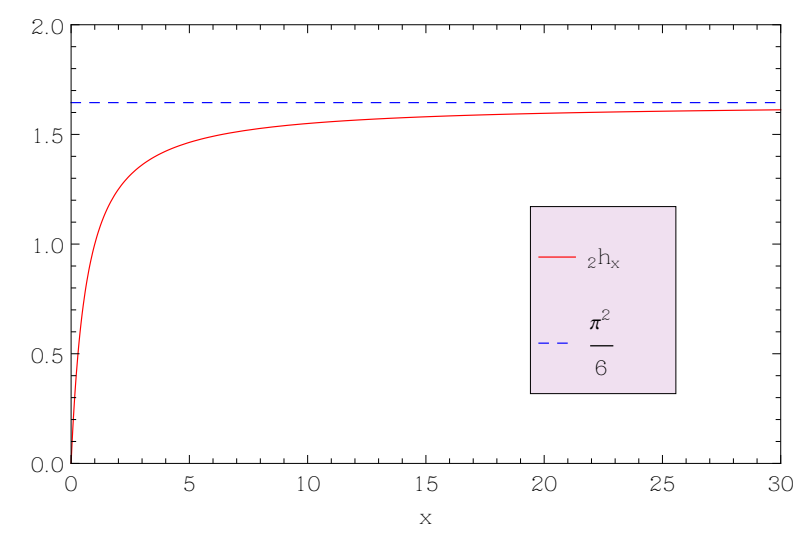

Figure 1. $2 h_{x}$ vs $x$ and $\lim _{x \rightarrow \infty} 2 h_{x}=\frac{\pi^{2}}{6}$.

The relevant extension to negative real indices will be considered later in the article.

Let us first consider the generating function associated with the second-order harmonic numbers, which can be cast in the form of an umbral exponential series, as follows. 
Definition 1. We introduce

$$
{ }_{2 h} e(t):=1+\sum_{n=1}^{\infty} \frac{t^{n}}{n !}\left({ }_{2} h_{n}\right)=e^{2} \hat{h} t \xi_{0}
$$

where ${ }_{2} \hat{h}$ is an umbral-like operator acting on the vaccum $\xi_{0}$, such that (see [10] for a complete treatment of the umbral method):

$$
\begin{aligned}
& { }_{2} \hat{h}^{n} \xi_{0}:=\xi_{n}={ }_{2} h_{n}, \quad n>0, \\
& { }_{2} \hat{h}^{0} \xi_{0}=1 \neq{ }_{2} h_{0}=0
\end{aligned}
$$

and

$$
{ }_{2} \hat{h}_{2}^{v} \hat{h}^{\mu} \xi_{0}={ }_{2} \hat{h}^{v+\mu} \xi_{0}, \quad \forall v, \mu \in \mathbb{R}
$$

(The action of the operator ${ }_{2} \hat{h}$ should be defined as explained in Reference [10]—namely, as the action of a shift operator on its vacuum, such as here $\xi_{0}$, in the following more rigorous way):

$$
\begin{aligned}
& { }_{2} \hat{h}:=e^{\partial_{z}} \\
& \left.{ }_{2} \hat{h}^{\mu} \xi_{0}=\left.e^{\mu \partial_{z}} \xi_{z}\right|_{z=0}=\left.\xi_{z+\mu}\right|_{z=0}=\left.\int_{0}^{1} \frac{1-x^{z+\mu}}{x-1} \ln (x) d x\right|_{z=0}=\int_{0}^{1} \frac{1-x^{\mu}}{x-1} \ln (x) d x .\right)
\end{aligned}
$$

Proposition 1. $\forall m \in \mathbb{N}$,

$$
{ }_{2 h} e(t, m):=\partial_{t_{2 h} h} e(t)={ }_{2} h_{m}+\sum_{n=1}^{\infty} \frac{t^{n}}{n !}\left({ }_{2} h_{n+m}\right)
$$

Proof. From Equations (6) and (7) it follows that, $\forall m \in \mathbb{N}$,

$$
{ }_{2 h} e(t, m)=\partial_{t}^{m}{ }_{2 h} e(t)=\partial_{t}^{m} e^{2} \hat{h} t \xi_{0}={ }_{2} \hat{h}^{m} e^{2} \hat{h} t \xi_{0}={ }_{2} h_{m}+\sum_{n=1}^{\infty} \frac{t^{n}}{n !}\left({ }_{2} h_{n+m}\right) .
$$

Corollary 1. Limiting ourselves to the first derivative only, it appears evident that the generating function (6) satisfies the identity

$$
\left\{\begin{array}{l}
\partial_{t_{2 h}} e(t)={ }_{2 h} e(t)+f_{2}(t), \\
{ }_{2 h} e(0)=1
\end{array}\right.
$$

where

$$
\begin{aligned}
& f_{2}(t)=\sum_{n=1}^{\infty} \frac{t^{n}}{(n+1)(n+1) !}=\frac{1}{t} \int_{0}^{t} \frac{e^{s}-s-1}{s} d s=-\frac{\operatorname{Ein}(-t)+t}{t} \\
& \operatorname{Ein}(z)=\int_{0}^{z} \frac{1-e^{-\zeta}}{\zeta} d \zeta .
\end{aligned}
$$

Observation 1. The problem of specifying the generating function of second-order harmonic numbers is reduced to the solution of the first-order differential Equation (10). The solution writes

$$
{ }_{2 h} e(t)=e^{t}\left(1+\sum_{n=1}^{\infty} \frac{1}{(n+1)^{2}}\left(1-e^{-t} e_{n}(t)\right)\right)
$$

where

$$
e_{n}(x)=\sum_{r=0}^{n} \frac{x^{r}}{r !}
$$


are the truncated exponential polynomials [16]. They belong to the family of Appél type polynomials [13] and are defined through the operational identity $[17,18]$ :

$$
e_{n}(x)=\frac{1}{1-\partial_{x}} \frac{x^{n}}{n !}
$$

Corollary 2. We can further elaborate on the previous identities (Equations (13) and (14)), and set

$$
\begin{aligned}
& \sum_{n=1}^{\infty} \frac{e_{n}(t)}{(n+1)^{2}}=Q_{2}(t), \\
& Q_{2}(t)=\frac{1}{1-\partial_{t}} f_{2}(t) .
\end{aligned}
$$

Furthermore, since

$$
\sum_{n=1}^{\infty} \frac{1}{(n+1)^{2}}=\frac{\pi^{2}}{6}-1
$$

we end up with

$$
\begin{aligned}
& { }_{2 h} e(t)=e^{t} \Sigma_{2}(t), \\
& \Sigma_{2}(t)=\frac{\pi^{2}}{6}-Q_{2}(t) e^{-t} .
\end{aligned}
$$

This new result can be viewed as an extension of the generating function for the first-order harmonic numbers derived by Gosper (see below) [19].

It is furthermore evident that the formalism allows the straightforward derivation of other identities, such as

Lemma 1. $\forall t \in \mathbb{R}$

$$
\sum_{n=1}^{\infty} \frac{t^{n}}{n !}\left({ }_{2} h_{n+m}\right)=e^{t} \sum_{s=0}^{m}\left(\begin{array}{c}
m \\
s
\end{array}\right) \Sigma_{2}^{(s)}(t)-{ }_{2} h_{m}
$$

where the upper index (s) denotes a s-order derivative and is a direct consequence of the identity in Equation (9).

The extension to higher-order harmonic numbers with $m>2$ follows the same logical steps-namely, the derivation of the associated Cauchy problem.

Corollary 3. Let $\forall t \in \mathbb{R}, \forall p \in \mathbb{N}: p>1$,

$$
f_{p}(t)=\sum_{n=1}^{\infty} \frac{t^{n}}{(n+1)^{p-1}(n+1) !}
$$

and

$$
\left\{\begin{array}{l}
\partial_{t}\left({ }_{p h} e(t)\right)={ }_{p h} e(t)+f_{p}(t) \\
{ }_{p h} e(0)=1
\end{array},\right.
$$

a Cauchy problem. Then, we can write the solution as:

$$
{ }_{p h} e(t)=e^{t}\left(1+\sum_{n=1}^{\infty} \frac{1}{(n+1)^{p}}\left(1-e^{-t} e_{n}(t)\right)\right)
$$

or

$$
{ }_{p h} e(t)=e^{t} \Sigma_{p}(t),
$$


with

$$
\begin{aligned}
& \Sigma_{p}(t)=\zeta(p)-Q_{p}(t) e^{-t}, \\
& \zeta(p)=\sum_{n=1}^{\infty} \frac{1}{n^{p}} \\
& Q_{p}(t)=\sum_{n=1}^{\infty} \frac{1}{(n+1)^{p}} e_{n}(t)=\frac{1}{1-\partial_{t}} f_{p}(t) .
\end{aligned}
$$

The case $p=1$ should be treated separately, because the sum on the right-hand side of Equation (21) apparently diverges.

Observation 2. It is accordingly worth noting that, since

$$
f_{1}(t)=\sum_{n=1}^{\infty} \frac{t^{n}}{(n+1) !}=\frac{1}{t}\left(e^{t}-t-1\right)
$$

we find

$$
\begin{aligned}
& { }_{1 h} e(t)=e^{t}\left(1+\int_{0}^{t} \frac{1-(\tau+1) e^{-\tau}}{\tau} d \tau\right)=e^{t} \Sigma_{1}(t), \\
& \Sigma_{1}(t)=e^{-t}+\operatorname{Ein}(t),
\end{aligned}
$$

which is a restatement of the Gosper derivation of the generating function of first-order harmonic numbers.

Further comments on the role played by the functions $\Sigma_{p}(t)$ will be provided in the final section of the paper.

We conclude this introductory section with the inclusion of a further identity.

Definition 2. Here, we introduce higher-order harmonic number umbral polynomials (for $m=1$, see Definition 5 in Reference [7]):

$$
\begin{aligned}
& { }_{m} h_{n}(x):=\left(x+{ }_{m} \hat{h}\right)^{n} \xi_{0}=x^{n}+\sum_{s=1}^{n}\left(\begin{array}{l}
n \\
s
\end{array}\right) x^{n-s}{ }_{m} h_{s} \\
& { }_{m} h_{0}(x)=1
\end{aligned}
$$

The ${ }_{m} h_{n}(x)$ are introduced in umbral form in complete analogy with those associated with the Bernoulli polynomials. They belong to the Sheffer family, and the relevant properties can be studied by means of the techniques discussed in Reference [17].

It has already been noted that (see Equation (31) in Reference [7])

$$
{ }_{1} h_{n}(-1)=(-1)^{n}\left(1-\frac{1}{n}\right)
$$

which coincides with an analogous identity derived with Mathematica in Reference [20] and, within the present framework from the recurrence

$$
{ }_{1} h_{n+1}(x)=(x+1){ }_{1} h_{n}(x)+\sum_{s=1}^{n}\left(\begin{array}{l}
n \\
s
\end{array}\right) \frac{1}{(s+1)} x^{n-s}=(x+1){ }_{1} h_{n}(x)+n \int_{0}^{1} d y \int_{0}^{y}(x+z)^{n-1} d z .
$$

Analogous results can be derived for the higher-order case, as in

$$
{ }_{2} h_{n}(-1)=(-1)^{n}\left(1-\frac{{ }_{1} h_{n}}{n}\right),
$$


and can be further generalized.

\section{Harmonic Numbers and Integral Transforms}

The identities we have dealt with in the previous section can be further generalized if the umbral procedure is merged with other techniques, involving things such as methods of an operational nature.

Proposition 2. We note that an extension of identities of the type reported in Equation (9) is provided by the sum

$$
\sum_{n=0}^{\infty} \frac{t^{n}}{n !} n^{m} h_{n}=e^{t} \sum_{k=0}^{m}\left\{\begin{array}{l}
m \\
k
\end{array}\right\} t^{k} \sum_{s=0}^{k}\left(\begin{array}{l}
k \\
s
\end{array}\right) \Sigma_{p}^{(k)}(t)
$$

where $\left\{\begin{array}{l}n \\ k\end{array}\right\}$ are Stirling numbers of the second kind, namely

$$
\left\{\begin{array}{l}
n \\
k
\end{array}\right\}=\frac{1}{k !} \sum_{j=0}^{k}(-1)^{k-j} j^{n}\left(\begin{array}{l}
k \\
j
\end{array}\right) .
$$

Proof. The result in Equation (30) was obtained by merging the umbral formalism with identities of an operational nature. By noting that (see, e.g., Equation (18) in Reference [21] and Equation (1) in [22], and for a more general use of these numbers, see [23-25])

$$
\left(x \partial_{x}\right)^{n}=\sum_{k=0}^{n}\left\{\begin{array}{l}
n \\
k
\end{array}\right\} x^{k} \partial_{x}^{k}
$$

and that if the following sum

$$
\sum_{n=0}^{\infty}\left(t^{n} a_{n}\right)=\sigma(t)
$$

does exist, then the following relation holds true

$$
\sum_{n=0}^{\infty} n^{m}\left(t^{n} a_{n}\right)=\left(t \partial_{t}\right)^{m} \sum_{n=0}^{\infty}\left(t^{n} a_{n}\right)=\left(t \partial_{t}\right)^{m} \sigma(t)=\sum_{k=0}^{m}\left\{\begin{array}{l}
m \\
k
\end{array}\right\} t^{k} \sigma^{(k)}(t) .
$$

The result contained in Equation (30) is, therefore, a consequence of Equations (9) and (34).

Furthermore, we noticed that by combining the umbral, Laplace transform, and integral representation methods, we could make further progress.

Example 2. Let us therefore note that (see Corollary 1 in [7])

$$
\frac{1}{1-{ }_{1} \hat{h} t} \xi_{0}=\sum_{r=1}^{\infty}{ }_{1} h_{r} t^{r}+1, \quad|t|<1 .
$$

The use of the Laplace transform allowed us to write the left-hand side of Equation (35) in the form of

$$
\frac{1}{1-{ }_{1} \hat{h} t} \xi_{0}=\int_{0}^{\infty} e^{-s} e^{s_{1} \hat{h} t} d s \xi_{0},
$$

which, on account of Equation (25), allows the conclusion controlla se l'eq e' la 25 


$$
\begin{aligned}
& 1+\sum_{n=1}^{\infty} t^{n}\left({ }_{1} h_{n}\right)=1-\frac{\ln (1-t)}{1-t}=1+\frac{L i_{1}(t)}{1-t}, \quad|t|<1, \\
& L i_{m}(x)=\sum_{r=1}^{\infty} \frac{x^{r}}{r^{m}} \equiv \text { Polylogarithm. }
\end{aligned}
$$

The same procedure applied to higher-order harmonic numbers yields the generating functions:

$$
\sum_{r=1}^{\infty}{ }_{m} h_{r} t^{r}+1=1+\frac{L i_{m}(t)}{1-t}, \quad|t|<1,
$$

already known for the case $m=2$.

The following example further underscores the versatility of the procedure we have proposed. We will indeed show that the use of the Gaussian identity

$$
e^{b^{2}}=\frac{1}{\sqrt{\pi}} \int_{-\infty}^{\infty} e^{-\tilde{\xi}^{2}+2 b \tilde{}} d \xi
$$

can be exploited to infer further identities on the properties of harmonic numbers.

Example 3. Now, we consider the following generating function

$$
{ }_{2 h} e_{2}(t):=1+\sum_{n=1}^{\infty} \frac{t^{n}}{n !}\left({ }_{2} h_{2 n}\right) .
$$

According to our formalism, the corresponding r.h.s. can be written as

$$
{ }_{2 h} e_{2}(t)=e^{\left({ }^{2} \hat{h}^{2}\right) t} \xi_{0},
$$

which, on account of the identity (39), can be written as

$$
{ }_{2} e_{2}(t)=\frac{1}{\sqrt{\pi}} \int_{-\infty}^{+\infty} e^{-s^{2}+2 s \sqrt{t} \hat{h}_{2}} d s \xi_{0} .
$$

The derivation of the sum in Equation (40) is therefore reduced to the evaluation of the following integral

$$
{ }_{2 h} e_{2}(t)=\frac{1}{\sqrt{\pi}} \int_{-\infty}^{+\infty} e^{-s^{2}}\left(\frac{\pi^{2}}{6} e^{2 s \sqrt{t}}-Q_{2}(2 s \sqrt{t})\right) d s=\frac{\pi^{2}}{6} e^{t}-q_{2}(t),
$$

with

$$
q_{2}(t)=\frac{1}{\sqrt{\pi}} \sum_{n=1}^{\infty} \frac{1}{(n+1)^{2}} \int_{-\infty}^{+\infty} e^{-s^{2}} e_{n}(2 s \sqrt{t}) d s
$$

\section{Final Comments}

Before concluding this paper, we show that the umbral formalism we have employed can be pushed even further to infer new properties of the harmonic numbers. To emphasise this point, we start from certain identities established in Reference [20] by the use of the Mathematica code, SIGMA. 
Among the examples discussed in Equation (18) in Reference [20], we pick out the following two:

$$
\begin{aligned}
& \text { 1) } \sum_{r=1}^{n}\left(\begin{array}{l}
n \\
r
\end{array}\right) \frac{(-1)^{r}}{r}=-{ }_{1} h_{n}, \\
& \text { 2) } \sum_{r=1}^{n}\left(\begin{array}{l}
n \\
r
\end{array}\right) \frac{(-1)^{r}}{r}{ }_{1} h_{r}=-{ }_{2} h_{n}
\end{aligned}
$$

We can transform the left-hand side of Equations (45) in a Newton binomial by an appropriate definition of umbra.

\section{Case 1}

Regarding the first, we define the operator [7]:

$$
\begin{aligned}
& \hat{\kappa}^{r} \psi_{0}=\frac{1}{r}, \quad r>0, \\
& \hat{\kappa}^{0} \psi_{0}=1,
\end{aligned}
$$

thus casting the first of Equation (45) in the form (see Equation (32) in Reference [7]):

$$
{ }_{1} h_{n}=1-(1-\hat{\kappa})^{n} \psi_{0}
$$

which can be exploited to once more derive the Gosper generating function. By multiplying both sides of Equation (47) by $\frac{t^{n}}{n !}$, and then by summing up on the index $n$, we obtain:

$$
\sum_{n=0}^{\infty} \frac{t^{n}}{n !} h_{n}=e^{t}\left(1-e^{-\hat{\kappa} t}\right)
$$

Keeping the m-th derivative with respect to both sides of Equation (48) yields:

$$
\sum_{n=0}^{\infty} \frac{t^{n}}{n !}{ }_{1} h_{n+m}=e^{t} \sum_{r=0}^{m}\left(\begin{array}{c}
m \\
r
\end{array}\right) \partial_{t}^{r} \phi(t)
$$

where

$$
\begin{aligned}
& \phi(t)=1-e^{-\hat{\kappa} t} \psi_{0} \\
& \partial_{t}^{r} \phi(t)=-(-\hat{\kappa})^{r} e^{-\hat{\kappa} t} \psi_{0}=-\sum_{s=0}^{\infty} \frac{(-1)^{s+r} \hat{\kappa}^{s+r}}{s !} t^{s} \psi_{0}= \begin{cases}-\sum_{s=0}^{\infty} \frac{(-1)^{s+r}}{s !(s+r)} t^{s}, & r>0, \\
-\sum_{r=1}^{\infty} \frac{-t^{r}}{r ! r}, & r=0 .\end{cases}
\end{aligned}
$$

\section{Case 2}

An analogous procedure can be exploited to handle the second:

$$
\hat{\mu}^{r} \eta_{0}=\frac{{ }_{1} h_{r}}{r}
$$

which allows for the derivation of the following identity

$$
\sum_{n=0}^{\infty} \frac{t^{n}}{n !} 2 h_{n+m}=e^{t} \sum_{r=0}^{m}\left(\begin{array}{c}
m \\
r
\end{array}\right) \partial_{t 2}^{r} \phi(t),
$$


with

$$
\begin{aligned}
& { }_{2} \phi(t)=1-e^{-\hat{\mu} t} \eta_{0}, \\
& \partial_{t 2}^{r} \phi(t)= \begin{cases}-\sum_{s=0}^{\infty} \frac{(-1)^{s+r}}{s !(s+r)} 1 h_{s+r} t^{s}, & r>0, \\
-\sum_{r=1}^{\infty} \frac{(-t)^{r}{ }_{1} h_{r}}{r ! r}, & r=0 .\end{cases}
\end{aligned}
$$

Remark 1. Let us now use the obvious identity (which holds for all operators):

$$
1^{n}=[(1-\hat{\mu})+\hat{\mu}]^{n} \eta_{0}
$$

Expanding the Newton binomial, we find

$$
\begin{aligned}
{[(1-\hat{\mu})+\hat{\mu}]^{n} \eta_{0} } & =\sum_{r=0}^{n}\left(\begin{array}{l}
n \\
r
\end{array}\right) \hat{\mu}^{n-r} \sum_{s=0}^{r}\left(\begin{array}{l}
r \\
s
\end{array}\right)(-1)^{s} \hat{\mu}^{s} \eta_{0}= \\
& =\left(\sum_{r=0}^{n-1}\left(\begin{array}{l}
n \\
r
\end{array}\right) \sum_{s=1}^{r}\left(\begin{array}{l}
r \\
s
\end{array}\right)(-1)^{s} \hat{\mu}^{n+s-r}+\sum_{r=0}^{n-1}\left(\begin{array}{l}
n \\
r
\end{array}\right) \hat{\mu}^{n-r}+\sum_{s=1}^{n}\left(\begin{array}{l}
n \\
s
\end{array}\right)(-1)^{s} \hat{\mu}^{s}+1\right) \eta_{0} .
\end{aligned}
$$

Remark 2. Choosing, for example, the first operator (46) and the property $\hat{\kappa}^{r} \hat{\kappa}^{s} \psi_{0}=\hat{\kappa}^{s+r} \psi_{0}=\frac{1}{s+r}$, we can finally elaborate (54) to get (see [24]):

$$
\sum_{r=1}^{n}\left(\begin{array}{l}
n \\
r
\end{array}\right) \frac{1}{r}=-\sum_{k=1}^{n}\left(\begin{array}{l}
n \\
k
\end{array}\right) \sum_{r=1}^{k}\left(\begin{array}{l}
k \\
r
\end{array}\right)(-1)^{r} \frac{1}{n+r-k}
$$

while when repeating the procedure with the realization (51), we end up with

$$
\sum_{r=1}^{n}\left(\begin{array}{l}
n \\
r
\end{array}\right) \frac{1 h_{r}}{r}=-\sum_{k=1}^{n}\left(\begin{array}{l}
n \\
k
\end{array}\right) \sum_{r=1}^{k}(-1)^{r}\left(\begin{array}{l}
k \\
r
\end{array}\right) \frac{1 h_{n+r-k}}{n+r-k}
$$

According to Reference [6] (see Equation (2)), it might also beconvenient to use the following umbral definition for the inverse of an integer.

Definition 3. We introduce the umbral operator

$$
\hat{a}^{n} \gamma_{0}:=\frac{1}{n+1}, \quad \forall n \in \mathbb{N}
$$

Proposition 3. $\forall n \in \mathbb{N}$ (see Equation 1 in [6])

$$
\frac{1}{n+1}=\sum_{s=0}^{n}\left(\begin{array}{l}
n \\
s
\end{array}\right)(-1)^{s} \frac{1}{s+1}
$$

Proof. It can be proved by induction that from (For $n=2$ we find $\hat{a}^{2} \gamma_{0}=\left(1-2 \hat{a}+\hat{a}^{2}\right) \gamma_{0}$, according to the prescription in Equation (58), we get

$$
\frac{1}{2+1}=1-2 \frac{1}{1+1}+\frac{1}{2+1}
$$

for $n=3, \hat{a}^{3} \gamma_{0}=\left(1-3 \hat{a}+3 \hat{a}^{2}-\hat{a}^{3}\right) \gamma_{0}$, we get

$$
\left.\frac{1}{4}=1-3 \frac{1}{1+1}+3 \frac{1}{2+1}-\frac{1}{3+1} .\right)
$$




$$
\hat{a}^{n} \gamma_{0}=(1-\hat{a})^{n} \gamma_{0}
$$

Furthermore, by using the obvious relation

$$
\hat{a}^{n} \gamma_{0}=[1-(1-\hat{a})]^{n} \gamma_{0}=\sum_{s=0}^{n}\left(\begin{array}{l}
n \\
s
\end{array}\right)(-1)^{s}(1-\hat{a})^{s},
$$

we end up with Equation (59).

We consider now the square of harmonic numbers, $\left[{ }_{1} h_{n}\right]^{2}$.

Definition 4. We introduce the umbral operator

$$
{ }_{1} \hat{h}_{(2)(2)}^{n} \tilde{\xi}_{0}:=\tilde{h}^{n} \tilde{\xi}_{0}=\left[{ }_{1} h_{n}\right]^{2}, \quad \forall n \in \mathbb{N} .
$$

Definition 5. The umbral operator (62) can be exploited to define the formal series:

$$
{ }_{1} h_{(2)} e(t):=\tilde{e}(t)=1+\sum_{n=1}^{\infty} \frac{t^{n}}{n !} \tilde{h}^{n} \tilde{\xi}_{0}=e^{\tilde{h} t} \tilde{\xi}_{0},
$$

specifying the associated generating function.

The derivation of Equation (63), according to the previously foreseen method, reduces to the solution of a first-order differential Equation, as it has been shown in the following example.

Example 4. By noting that

$$
\tilde{h}^{n+1} \tilde{\xi}_{0}=\left[{ }_{1} h_{n}\right]^{2}+2 \frac{{ }_{1} h_{n}}{n+1}+\frac{1}{(n+1)^{2}}
$$

and that

$$
\partial_{t} \tilde{e}(t)=\tilde{h} e^{\tilde{h} t} \tilde{\xi}_{0}
$$

we easily find

$$
\partial_{t} \tilde{e}(t)=\tilde{e}(t)-1+\frac{2}{t} \sum_{n=0}^{\infty} \frac{t^{n+1}}{(n+1) !}\left({ }_{1} h_{n+1}-\frac{1}{n+1}\right)+\sum_{n=0}^{\infty} \frac{t^{n}}{(n+1)(n+1) !}
$$

or

$$
\partial_{t} \tilde{e}(t)=\tilde{e}(t)+\frac{2}{t}\left({ }_{1} h e(t)-t-1\right)-\frac{1}{t} \int_{0}^{t} \frac{e^{s}-s-1}{s} d s
$$

which can be solved by the use of the same technique as before, made only slightly more complicated by the non-homogeneous term. The Equation can be straightforwardly solved, and the relevant solution, for $\tilde{e}(0)=1$, reads

$$
\tilde{e}(t)=e^{t}(1+\chi(t)),
$$

where

$$
\begin{aligned}
& \chi(t)=\int_{0}^{t} e^{-s} \beta(s) d s, \\
& \beta(t)=\frac{2}{t}\left({ }_{1} h e(t)-t-1\right)-f_{2}(t) .
\end{aligned}
$$

Remark 3. In the introductory section we mentioned the link between harmonic and Bernoulli numbers, and they have been paradigmatic examples of applications of umbral methods. Regarding the finite sum of power of integers, we find, for example (according to Chapter 6 in Reference [4]), 


$$
\sum_{r=0}^{n} r^{m}={ }_{(-m)} h_{n}=\frac{1}{m+1}\left[(\hat{b}+n+1)^{m+1}-\hat{b}^{m+1}\right] \theta_{0}
$$

where

$$
\hat{b}^{r} \theta_{0}=B_{r}
$$

is the umbral operator which provides Bernoulli numbers $B_{r}$.

Proposition 4. The use of Equation (70) yields the generating function:

$$
\sum_{m=0}^{\infty} \frac{(-m) h_{n} z^{m+1}}{m !}=\frac{z}{e^{z}-1}\left(e^{(n+1) z}-1\right), \quad \forall z \in \mathbb{R}:|z|<2 \pi .
$$

Proof. $\forall z \in \mathbb{R}:|z|<2 \pi$

$$
\begin{aligned}
\sum_{m=0}^{\infty} \frac{(-m) h_{n} z^{m+1}}{m !} & =\sum_{m=0}^{\infty} \frac{\left[(\hat{b}+n+1)^{m+1}-\hat{b}^{m+1}\right] z^{m+1}}{(m+1) !} \theta_{0}=\left(e^{(\hat{b}+n+1) z}-e^{\hat{b} z}\right) \theta_{0}= \\
& =\left(e^{(n+1) z}-1\right) e^{\hat{b} z} \theta_{0}=\left(e^{(n+1) z}-1\right) \sum_{r=0}^{\infty} \frac{z^{r}}{r !} \hat{b}^{r} \theta_{0}= \\
& =\left(e^{(n+1) z}-1\right) e^{\hat{b} z} \theta_{0}=\left(e^{(n+1) z}-1\right) \sum_{r=0}^{\infty} \frac{z^{r}}{r !} B_{r}
\end{aligned}
$$

which, by taking into account that the generating function of Bernoulli numbers is

$$
\sum_{r=0}^{\infty} \frac{z^{r}}{r !} B_{r}=\frac{z}{e^{z}-1}
$$

yields the statement in Equation (72).

In this paper, we have provided a hint of the possible interplay between the theory of harmonic numbers and operational methods (be they of umbral ordinary nature). Further progress will be presented in future investigations.

Author Contributions: Conceptualization, G.D.; methodology, G.D., S.L.; data curation: S.L.; validation, G.D., S.L., E.S., H.M.S.; formal analysis, G.D., S.L., E.S., H.M.S.; writing-riginal draft preparation: G.D.; writing-review and editing: S.L.

Funding: The work of S.L. was supported by a Enea-Research Center Individual Fellowship

Acknowledgments: The Authors recognizes the kind help of the Referees who pushed for a better organized presentation and for including in the study the Bernoulli numbers.

Conflicts of Interest: The authors declare no conflict of interest.

\section{References}

1. Roman, S. The Umbral Calculus; Academic Press: New York, NY, USA, 1984; pp. 93-100.

2. Dattoli, G.; Cesarano, C.; Lorenzutta, S. Bernoulli numbers and polynomials from a more general point of view. Rendiconti Matematica 2002, 22, 193-202.

3. Dattoli, G.; Migliorati, M. Operational Methods, Harmonic Numbers and Riemann Function; Internal Report Enea Frascati (Rome) RT/2008/29/FIM; Edizioni Scientifiche—ENEA Centro Ricerche Frascati: Rome, Italy, 2008.

4. Graham, R.L.; Knuth, D.E.; Patashnik, O. Concrete Mathematics: A Foundation for Computer Science, 2nd ed.; Addison-Wesley Longman Publishing Co., Inc.: Boston, MA, USA, 1994.

5. Dattoli, G.; Srivastava, H.M. A Note on Harmonic Numbers, Umbral Calculus and Generating Functions. Appl. Math. Lett. 2008, 21, 686-693. [CrossRef]

6. Dattoli, G.; Zhukovsky, K. Umbral Methods, Combinatorial Identities and Harmonic Numbers. Appl. Math. 2011, 1, 46-49. 
7. Dattoli, G.; Germano, B.; Licciardi, S.; Martinelli, M.R. Umbral Methods and Harmonic Numbers. Axioms 2018, 7, 62.

8. Coffey, M.W. Expressions for harmonic humber exponential generating functions. In Contemporary Mathematics, Gems in Experimental Mathematics; Amdeberhan, T., Simos, E.T., Moll, V.H., Eds.; American Mathematical Society: Washington, DC, USA, 2009; Volume 517, pp. 113-126.

9. Cvijović, D. The Dattoli-Srivastava Conjectures Concerning Generating Functions Involving the Harmonic Numbers. Appl. Math. Comput. 2010, 215, 4040-4043. [CrossRef]

10. Licciardi, S. Umbral Calculus, a Different Mathematical Language. arXiv 2018, arXiv:1803.03108.

11. Choi, J. Finite summation formulas involving binomial coefficients, harmonic numbers and generalized harmonic numbers. J. Inequal. Appl. 2013, 49, 11. [CrossRef]

12. Sofo, A.; Srivastava, H.M. A family of shifted harmonic sums. Ramanujan J. 2015, 37, 89-108. [CrossRef]

13. Sofo, A.; Srivastava, H.M. dentities for the harmonic numbers and binomial coefficients. Ramanujan J. 2011, 25, 93-113. [CrossRef]

14. Choi, J.; Srivastava, H.M. Some summation formulas involving harmonic numbers and generalized harmonic numbers. Math. Comput. Model. 2011, 54, 2220-2234. [CrossRef]

15. Mezo, I. Exponential Generating Function of Hyper-Harmonic Numbers Indexed by Arithmetic Progressions. Cent. Eur. J. Math. 2013, 11, 931-939.

16. Dattoli, G.; Ricci, P.E.; Marinelli, L. Generalized Truncated Exponential Polynomials and Applications, in: Rendiconti dell'Istituto di Matematica dell'Università di Trieste. Int. J. Math. 2002, 34, 9-18.

17. Dattoli, G.; Migliorati, M.; Srivastava, H.M. Sheffer polynomials, monomiality principle, algebraic methods and the theory of classical polynomials. Math. Comput. Model. 2007, 45, 1033-1041. [CrossRef]

18. Dattoli, G.; Sabia, E. Generalized Transforms and Special functions. arXiv 2010, arXiv:1010.1679.

19. Gosper R.W. Harmonic summation and exponential gfs. math-fun@cs.arizona.edu posting, 2 August 1996. In Harmonic Number. MathWorld—A Wolfram Web Resource; Sondow, J., Weisstein, E.W., Eds.; 1996; Available online: http:/ / mathworld.wolfram.com/HarmonicNumber.html (accessed on 1 May 2019).

20. Schmidt, M.D. Zeta Series Generating Function Transformations Related to Polylogarithm Functions and the k-Order Harmonic Numbers. Online J. Anal. Comb. 2017, 12, 2.

21. Dattoli, G.; Germano, B.; Martinelli, M.R.; Ricci, P.E. Touchard like polynomials and generalized Stirling numbers. Appl. Math. Comput. 2012, 218, 6661-6665. [CrossRef]

22. Lang, W. On Generalizations of the Stirling Number Triangles. J. Integer. Seq. 2000, 3, 3.

23. Srivastava, H.M.; Manocha, H.L. A Treatise on Generating Functions; Halsted Press (Ellis Horwood Limited, Chichester): Chichester, UK; John Wiley and Sons: New York, NY, USA, 1984.

24. Comtet, L. Advanced combinatorics: The Art of Finite and Infinite Expansions; Springer: Berlin/Heidelberg, Germany, 1974.

25. Johnson, W.P. The Curious History of Faá di Bruno's Formula. Am. Math. Mon. 2002, 109, 217-234.

(C) 2019 by the authors. Licensee MDPI, Basel, Switzerland. This article is an open access article distributed under the terms and conditions of the Creative Commons Attribution (CC BY) license (http:/ / creativecommons.org/licenses/by/4.0/). 